\title{
Paradoxical emboli: the proof was short lived
}
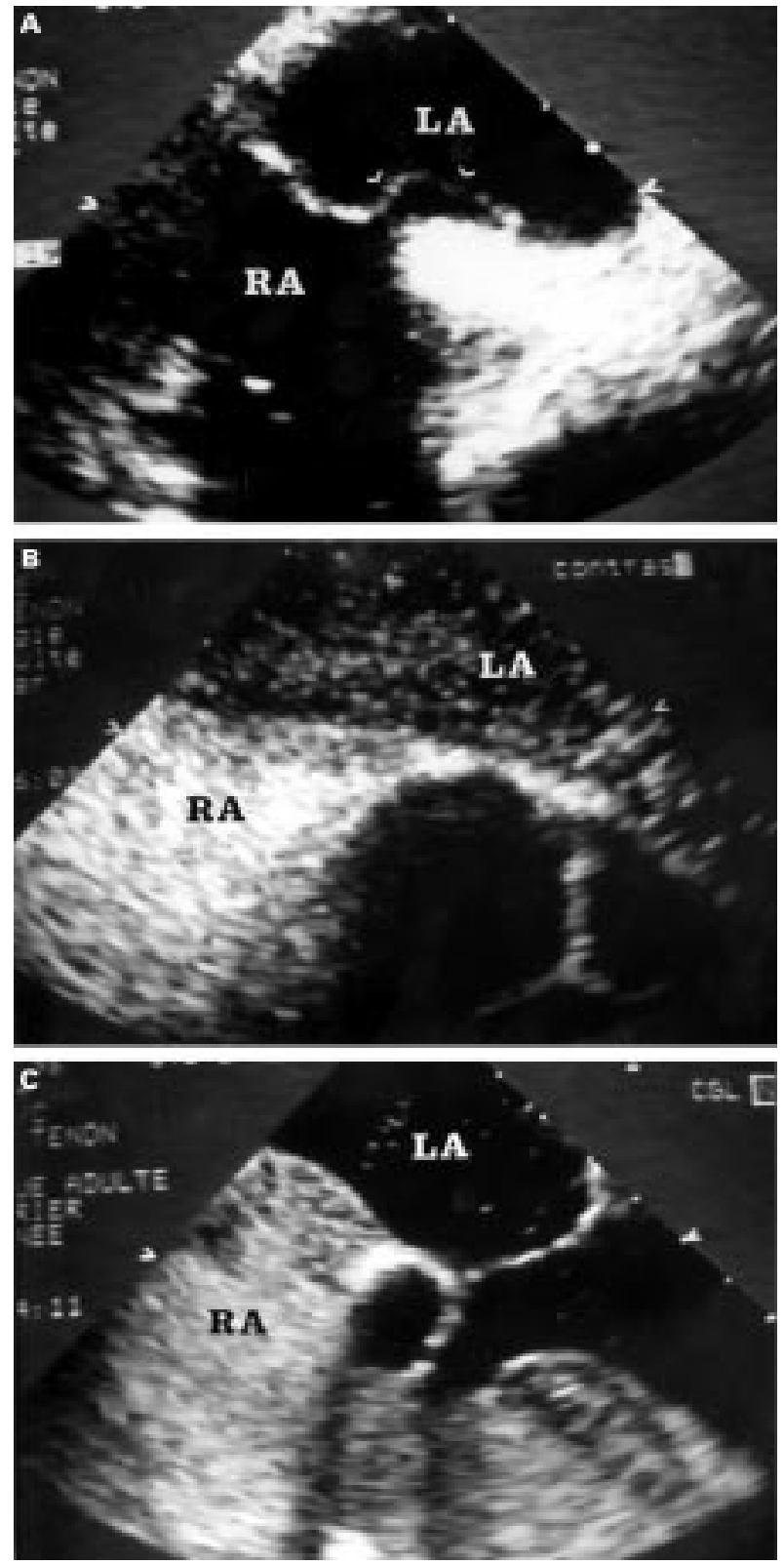

A 76 year old woman was referred with a right hemiplegia. She was on treatment for hypertension, angina pectoris, and dyslipidaemia. She had been complaining of pain in the right leg and increasing exertional dyspnoea for two days when she suddenly experienced right sided weakness. On examination she was polypneic and cyanotic; she had right hemiplegia and aphasia which were related to a deep infarct in the territory of the left middle cerebral artery on CT. Arterial blood gas analysis showed hypoxia and hypocapnia, and radionuclide lung imaging confirmed bilateral pulmonary emboli. Venous ultrasonography detected a right sural vein thrombosis. Carotid and transcranial Doppler ultrasonography were normal. A first contrast transesophageal echocardiography disclosed a moderate atrial septal aneurysm (figure, A arrowhead) and a massive shunt (figure, B) between the right atrium (RA) and the left atrium (LA) associated with pulmonary arterial hypertension, suggesting that paradoxical emboli might explain the cerebral infarction. Complete coagulation studies, including resistance to activated $\mathrm{C}$ protein, were negative. Intravenous heparin and oxygen were administered, followed by warfarin and the patient's pulmonary symptoms fully resolved.Three weeks later radionuclide lung imaging was normal and contrast transoesophageal echocardiography disclosed no significant right to left shunt or pulmonary arterial hypertension (figure,C). One year later she remained severely disabled.

\section{O HEINZLEF \\ B CORMIER \\ D MEYNIEL \\ Tenon Hospital, Paris, France}

Correspondence to: Dr Olivier Heinzlef, Department of Neurology, Tenon Hospital, 4 rue de la Chine, 75020 Paris, France. Telephone 003356016109 ; fax 0033156017005 ; email olivier.heinzlef@tnn.ap-hop-paris.fr 